Chapman University

Chapman University Digital Commons

ESI Working Papers

Economic Science Institute

$11-13-2019$

\title{
Cooperation in Indefinitely Repeated Helping Games: Existence and Characterization
}

\author{
Gabriele Camera \\ Chapman University, camera@chapman.edu
}

Alessandro Gioffré

Univeristy of Basel

Follow this and additional works at: https://digitalcommons.chapman.edu/esi_working_papers

Part of the Econometrics Commons, Economic Theory Commons, and the Other Economics Commons

\section{Recommended Citation}

Camera, G., \& Gioffré, A. (2019). Cooperation in indefinitely repeated helping games: existence and characterization. ESI Working Paper 19-28. https://digitalcommons.chapman.edu/esi_working_papers/ 288/

This Article is brought to you for free and open access by the Economic Science Institute at Chapman University Digital Commons. It has been accepted for inclusion in ESI Working Papers by an authorized administrator of Chapman University Digital Commons. For more information, please contact laughtin@chapman.edu. 


\section{Cooperation in Indefinitely Repeated Helping Games: Existence and Characterization}

\section{Comments}

ESI Working Paper 19-28

This paper later underwent peer review and was published as:

Camera, G., \& Gioffré, A. (2019). Cooperation in indefinitely repeated helping games: existence and characterization. Journal of Economic Behavior \& Organization, 200, 1344-1356. https://doi.org/10.1016/ j.jebo.2019.11.014 


\title{
Cooperation in indefinitely repeated helping games: existence and characterization*
}

\author{
Gabriele Camera \\ Alessandro Gioffré \\ ESI, Chapman University \\ University of Florence \\ University of Bologna
}

November 13, 2019

\begin{abstract}
Experiments that investigate the spontaneous emergence of money in laboratory societies rely on indefinitely repeated helping games with random matching (Camera et al., 2013; Camera and Casari, 2014). An important open issue is the lack of a general proof of existence of an equilibrium capable of supporting the efficient allocation under private monitoring, without money. Here, we fill this gap by offering a general proof, as well as by characterizing the efficient non-monetary equilibrium. This technique can be extended to study games with simultaneous actions.

Keywords: tacit coordination, random matching, social norms.

JEL codes: E4, E5, C7

\footnotetext{
* We would like to thank Co-Editor Cesar Martinelli and an anonymous referee for helpful comments and suggestions, as well as workshop and seminar participants at the University of Florence. Corresponding author: Gabriele Camera, Economic Science Institute, Chapman University, One University dr., Orange, CA 92866; Tel.: 714-628-2806; e-mail: camera@chapman.edu. Alessandro Gioffré, DISEI, University of Florence; Tel.:+39 055275 9606; e-mail: alessandro.gioffre@unifi.it
} 


\section{Introduction}

The two-sided Prisoner's Dilemma (PD) is the most common strategic framework used in the laboratory to study cooperation in indefinitely repeated games. This game has been adopted both to study cooperation among partners interacting in a fixed pair (Dal Bó and Fréchette, 2018), as well as in groups of strangers interacting in pairs that are randomly rematched in each round of play (Camera and Casari, 2009; Duffy and Ochs, 2009). In the former design, two subjects are placed in a fixed match at the start of the game and repeatedly play the PD game for an uncertain number of rounds. In the latter design, subjects from a fixed and even-numbered group of participants are randomly rematched in each round with uniform probability, to play the PD with a series of opponents whose identity and past actions are private information. This design choice is convenient to study interaction in environments where neither reciprocity nor reputation can be established, because it corresponds to the theoretical platform adopted in the social norms literature initiated by Kandori (1992) and Ellison (1994). That literature offers a general proof of existence of cooperative equilibrium for groups of any desired size; the theory also offers a characterization of off-equilibrium endogenous variables. These general theoretical results can be used to determine with a great deal of precision the set of experimental parameters that are (in)consistent with cooperative equilibrium; in particular, the size of the group, the probability of the game ending, and the various elements of the payoff matrix.

More recently, experiments have extended the investigation of cooperation in groups of strangers to situations in which the interaction is one-sided, being based 
on a "helping game" (Nowak, 2006). Here, only one player takes an action in each pair, and this is determined by random assignment: in each round, each player is randomly assigned both an opponent and a role in the pair (active or inactive); see Camera et al. (2013) and Camera and Casari (2014).

Moving from a two-sided to a one-sided stage game provides both opportunities and challenges for the experimentalist. An advantage is that it makes the intertemporal nature of cooperation apparent to subjects as each stage is effectively a dictator game. It also makes the design suitable to study the institution of monetary trade as a possible alternative to informal social norms (see the discussion in Bigoni et al., forthcoming). A challenge is that moving from a two-sided to a one-sided stage game fundamentally alters the environment if there is private monitoring, because the lower frequency of actions affects the way in which information about defections evolves off-equilibrium relative to a two-sided game. As a result, this alters off-equilibrium payoffs, and therefore the parameters supporting cooperative equilibrium in a manner that is opaque because the theoretical literature neither offers a general proof of existence nor a characterization of cooperative equilibrium for the case of private monitoring. It follows that the experimentalist has to establish existence of cooperative equilibrium case-by-case, for the given experimental group size, as done in Camera and Casari (2014) for example. ${ }^{1}$ Here we fill this important gap by generalizing the study of social norms and cooperation to indefinitely repeated helping games characterized by private monitoring

\footnotetext{
${ }^{1}$ The problem is not present if there is public monitoring, because in this case the group size no longer matters since off-equilibrium information about defections is immediately made available to the entire group (e.g., see Camera et al., 2013).
} 
and random matching.

There are two main contributions, with both substantive and methodological value. We derive sufficient conditions supporting full cooperation in sequential equilibrium for a general population size and a general payoff matrix. We also characterize the evolution of the key endogenous variables, such as payoffs and the spread of punishment, off equilibrium. As demonstrated below, these results can be readily applied to determine the parameters of a laboratory implementation. Furthermore, the technique we develop in terms of tracking the spread of information about defections off-equilibrium can be applied to other games in which actions are one-sided.

To understand how two-sided and one-sided game differ, let us start by recalling what emerges from the study of social norms when the game is a PD. The studies in Kandori (1992) and Ellison (1994) demonstrate that groups of strangers can attain the efficient, fully cooperative outcome if two factors are present. First, the payoff structure should provide incentives to cooperate if everyone else does the same. Second, players should be capable of tacitly coordinating on an irreversible punishment process leading to a progressive, contagious cooperation decline in the group, triggered by any privately observed defection. Sufficient conditions for these incentives to be present are (i) a low gain from defecting in meetings with cooperators (the "temptation payoff"), and (ii) a large cost from cooperating in meetings with defectors (the "sucker's payoff"). These two conditions are associated with two distinct threshold values for the player's discount factor $\delta$, denoted $\delta_{1}$ and $\delta_{2}$ where $\left[\delta_{1}, \delta_{2}\right] \neq \emptyset$. The first condition implies that $\delta$ must be sufficiently 
high, $\delta \geq \delta_{1}$, while the second implies that it must be sufficiently low, $\delta \leq \delta_{2}$. Full cooperation is an equilibrium for $\delta \in\left[\delta_{1}, \delta_{2}\right]$.

Here we show that moving from a two-sided PD game to the one-sided helping game alters the incentive to cooperate in equilibrium, and also to punish off equilibrium. Therefore both $\delta_{1}$ and $\delta_{2}$ are affected. Intuitively, in a helping game with random role assignment a player is passive at random points in time, and in each period half of the players are passive and half active. Off equilibrium, passive players cannot protect themselves from the possibility of meeting free-riders, by choosing to defect-something that instead can be done in the PD game. As a result, this influences the payoff associated to grim punishment off equilibrium, and therefore the incentive for an active player to move off-equilibrium by refusing to cooperate. We provide this intuition by fully characterizing the community punishment process, the off-equilibrium payoffs, and also the thresholds $\delta_{1}$ and $\delta_{2}$. An application with code to compute the critical equilibrium parameters is provided to facilitate experimental design (see online Supplementary Materials).

The study proceeds as follows. Section 2 describes the model and the grim strategy we focus on. Section 3 characterizes the contagious punishment process implied by the grim strategy. Section 4 reports the main result, while Section 5 provides an illustration through a simulation, which is then compared to results from an experiment. Section 6 concludes. 


\section{Model}

The model generalizes the baseline, no token design in Camera and Casari (2014) to any group size. $N=2 n \geq 4$ infinitely-lived players are arranged in consumerproducer pairs in each period using uniform random matching; roles are randomly assigned with equal probability in each round. Every pair plays a helping game in which the producer chooses between $C$ or $D$ ("cooperate," "defect"), with period payoffs reported below. ${ }^{2}$

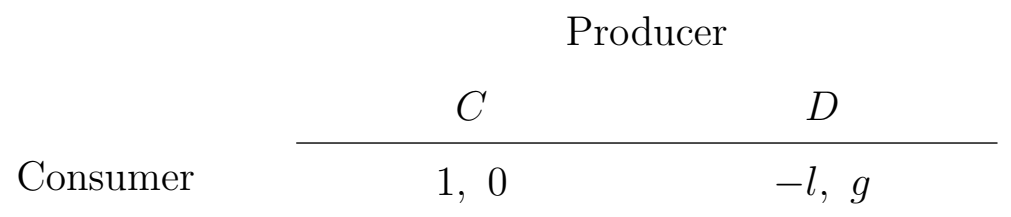

Figure 1: The stage game between a consumer and a producer.

Assume $g>0$ and $1>g-l$. Cooperation payoffs to consumer and producer are 1 and 0 . Note that $l$ can be positive or negative. If positive, then there is no restriction on the value it can take. However, if $l<0$ then the inequality above implies that $l>g-1$, that is $l$ cannot be too small or the surplus would be larger under the defection outcome than under cooperation.

Players have linear preferences and discount future payoffs with common factor $\delta \in(0,1)$. The ex-ante payoff (unconditional on the role) to player $i$ in the infinitely repeated game is

$$
\mathbb{E} \sum_{t=0}^{\infty} \delta^{t} \pi\left(a_{h, t}\right)
$$

Here, $\pi\left(a_{h, t}\right)$ denotes the payoff to player $i$ when meeting $-i$; $a_{h, t}=C, D$ is the ${ }^{2} \mathrm{~A}$ more detailed discussion of this game is in the Appendix A.1. 
action taken if the round is $t ; h$ is a random variable taking either value $i$ or $-i$ with equal probability, i.e., it selects the producer. In the efficient outcome, every producer cooperates in every meeting, so the ex-ante payoff is $v_{0}=\frac{1}{2(1-\delta)}$, while payoffs conditional on the role ( $c$ and $p$, consumer and producer) are

$$
v_{0}^{c}=1+\delta v_{0} \quad \text { and } \quad v_{0}^{p}=\delta v_{0} .
$$

There is private monitoring: players can only observe outcomes and actions in their pair and cannot see the history of play of their opponents. We will investigate if the efficient outcome is sustained in sequential equilibrium by a trigger strategy whereby a player can be in a cooperative state-where he chooses $C$ as a producer and is inactive as a consumer - or an irreversible punishment state, where he chooses $D$ as a producer and is inactive as a consumer.

Definition 1 (Trigger strategy). In round $t=0$, the player is in the cooperative state. In $t \geq 1$, the player remains in that state if he has not observed a defection; otherwise, the player switches to the punishment state.

This strategy encompasses two modes of behavior: cooperation, which corresponds to equilibrium play, and punishment, which corresponds to off-equilibrium play. The player starts by cooperating (if he is a producer in round 1) and keeps cooperating as long as he has experienced a fully cooperative outcome in every past round of play. The player punishes by choosing defection as a producer, if the player deviated by defecting or observed a deviation from equilibrium play. The strategy is a social norm if every player (tacitly) coordinates on using it. Because of private monitoring, off-equilibrium cooperators switch to punishment gradually, 
depending on the realization of random meetings and role assignment. We now study this community enforcement threat.

The strategy in Definition 1 is illustrated in Figure 2. Unlike the grim punishment strategy used in the PD game, the player who has switched from a cooperative to a punishment state will defect only in a random subsequence of rounds of the continuation game. The defection takes place at random intervals, $T_{1}, T_{2}, T_{3}, \ldots$ following the first observed defection, in the rounds when the player is a producer. In all other rounds the player is a consumer and has no action to take.

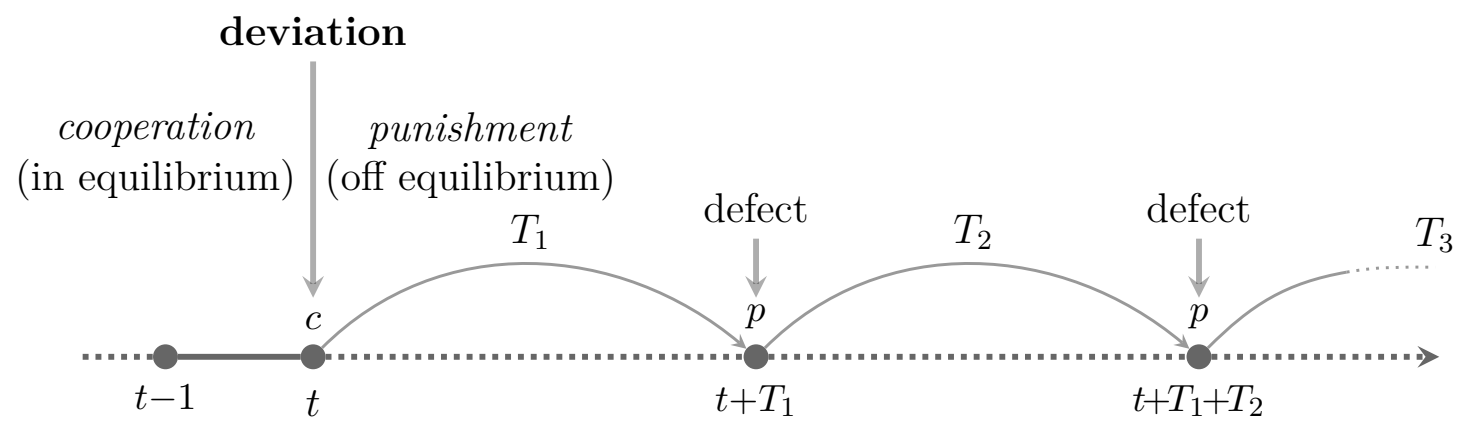

Figure 2: Punishment when producer and consumer roles are randomly assigned.

Notes: $c=$ the subject is a consumer, $p=$ the subject is a producer. In capital letters the number of periods in between two producer roles, where $T_{1}, T_{2}, T_{3}, \ldots \geq 1$ is the random length of the interval.

\section{The contagious punishment process}

Consider an even population of $M$ players. Off equilibrium, partition them into defectors (punishment state) and cooperators (cooperative state). Cooperators become defectors at random points in time, via a contagious process described by the $M \times M$ upper-triangular Markov matrix 


$$
\mathcal{Q}_{M}:=\left(\begin{array}{cccccccccc}
Q_{11} & Q_{12} & 0 & 0 & 0 & 0 & \ldots & 0 & 0 & 0 \\
0 & Q_{22} & Q_{23} & Q_{24} & 0 & 0 & \ldots & 0 & 0 & 0 \\
0 & 0 & Q_{33} & Q_{34} & Q_{35} & Q_{36} & \ldots & 0 & 0 & 0 \\
\vdots & \vdots & \vdots & \vdots & \vdots & \vdots & \ldots & \vdots & \vdots & \vdots \\
0 & 0 & 0 & 0 & 0 & 0 & \ldots & Q_{M-2, M-2} & Q_{M-2, M-1} & Q_{M-2, M} \\
0 & 0 & 0 & 0 & 0 & 0 & \ldots & 0 & Q_{M-1, M-1} & Q_{M-1, M} \\
0 & 0 & 0 & 0 & 0 & 0 & \ldots & 0 & 0 & 1
\end{array}\right)
$$

Suppose at the start of a round there are $k=1, \ldots, M$ defectors. Players are matched and roles are randomly assigned with equal probability. The probability to transition to $k^{\prime} \geq k$ defectors next round is:

$$
Q_{k k^{\prime}}(M)=\sum_{j=k^{\prime}-k}^{\min (k, M-k)} \lambda_{k j}\left(\begin{array}{c}
j \\
k^{\prime}-k
\end{array}\right)\left(\frac{1}{2}\right)^{j} .
$$

Here

$$
\lambda_{k j}(M):=\frac{j !\left(\begin{array}{c}
k \\
j
\end{array}\right)\left(\begin{array}{c}
M-k \\
j
\end{array}\right)(k-j-1) ! !(M-k-j-1) ! !}{(M-1) ! !}
$$

is the probability of having $j$ mixed matches (defector-cooperator), where

$$
j \in \begin{cases}\{0,2,4, \ldots, \min (k, M-k)\} & \text { if } k=\text { even } \\ \{1,3,5, \ldots, \min (k, M-k)\} & \text { if } k=\text { odd }\end{cases}
$$

Given $j$ mixed matches, the probability that $k^{\prime}-k \leq j$ defectors get a producer role is

$$
\left(\begin{array}{c}
j \\
k^{\prime}-k
\end{array}\right)\left(\frac{1}{2}\right)^{j}
$$

Summing over all possible numbers of mixed matches that can generate $k^{\prime}-k$ new defectors (i.e., $\left.k^{\prime}-k \leq j \leq \min (k, M-k)\right)$ gives us (2). The properties of this matrix are described in Camera and Gioffré (2014). 


\subsection{Continuation payoffs off equilibrium}

Let $M=N$ and $v_{k}$ denote the (ex-ante continuation) payoff to a generic defector, when $k=1, \ldots, N$ players are defectors (punishment state) and $N-k$ are cooperators (cooperation state). Due to uniform random matching, a defector meets a cooperator with probability $\sigma_{k}:=\frac{N-k}{N-1}$. Recursively define

$$
v_{k}=\sigma_{k} \frac{1+g}{2}+\left(1-\sigma_{k}\right) \frac{g-l}{2}+\delta \sum_{k^{\prime}=k}^{N} Q_{k k^{\prime}}(N) v_{k^{\prime}} \text {, }
$$

where $v_{k^{\prime}}$ is the payoff to a defector next round, when there are $k^{\prime} \geq k$ defectors.

Rearranging

$$
v_{k}=\frac{g-l}{2}+\frac{1+l}{2} \sigma_{k}+\delta \sum_{k^{\prime}=k}^{N} Q_{k k^{\prime}}(N) v_{k^{\prime}} .
$$

Letting $v:=\left(v_{1}, \ldots, v_{N}\right)^{\top}, \sigma:=\left(\sigma_{1}, \ldots, \sigma_{N}\right)^{\top}$, and $\mathbf{1}:=(1, \ldots, 1)^{\top}$ we have

$$
v=\frac{g-l}{2} \mathbf{1}+\frac{1+l}{2} \sigma+\delta \mathcal{Q}_{N} v \quad \Rightarrow \quad\left(\mathcal{I}_{N}-\delta \mathcal{Q}_{N}\right) v=\frac{g-l}{2} \mathbf{1}+\frac{1+l}{2} \sigma
$$

where $\mathcal{I}_{N}$ is the $N \times N$ identity matrix. Matrix $\mathcal{I}_{N}-\delta \mathcal{Q}_{N}$ is invertible and we have $\left(\mathcal{I}_{N}-\delta \mathcal{Q}_{N}\right)^{-1} \mathbf{1}=(1-\delta)^{-1} \mathbf{1}$, because each element of $\left(\mathcal{I}_{N}-\delta \mathcal{Q}_{N}\right)^{-1}$ is non-negative and its rows sum to $(1-\delta)^{-1}$. Therefore,

$$
v=\left(\mathcal{I}_{N}-\delta \mathcal{Q}_{N}\right)^{-1}\left(\frac{g-l}{2} \mathbf{1}+\frac{1+l}{2} \sigma\right)=\frac{g-l}{2(1-\delta)} \mathbf{1}+\frac{1+l}{2}\left(\mathcal{I}_{N}-\delta \mathcal{Q}_{N}\right)^{-1} \sigma
$$

Hence:

$$
v_{k}=\frac{g-l}{2(1-\delta)}+\frac{1+l}{2(1-\delta)} \phi_{k}(\delta)
$$

with $\phi_{k}(\delta):=(1-\delta) e_{k}^{\top}\left(\mathcal{I}_{N}-\delta \mathcal{Q}_{N}\right)^{-1} \sigma$ and $e_{k}$ is the $N$-dimensional column vector with 1 in the $k^{\text {th }}$ position and 0 everywhere else. 
We present two lemmas and then the main result.

Lemma 1. For $k=1, \ldots, N-1, \phi_{k}(\delta)$ falls in $\delta \in(0,1)$.

Proof. In Appendix.

Lemma 2. For all $\delta \in[0,1)$ we have

$$
\frac{\delta}{2(1-\delta)}\left[\phi_{1}(\delta)-\phi_{2}(\delta)\right]=1-\phi_{1}(\delta)
$$

Proof. In Appendix.

It is convenient to split defector $i$ 's payoff in two parts depending on whether he meets a cooperator or a defector:

$$
\begin{aligned}
& v_{k}=\sigma_{k}\{\frac{1}{2} \overbrace{\left[1+\delta \sum_{k^{\prime}=k-1}^{N-2} Q_{k-1, k^{\prime}}(N-2) v_{k^{\prime}+1}\right]}^{i \text { is a consumer }}+\frac{1}{2} \overbrace{\left[g+\delta \sum_{k^{\prime}=k-1}^{N-2} Q_{k-1, k^{\prime}}(N-2) v_{k^{\prime}+2}\right]}^{i \text { is a producer }}\} \\
& +\left(1-\sigma_{k}\right)\{\frac{1}{2} \overbrace{\left.\left[-l+\delta \sum_{k^{\prime}=k-2}^{N-2} Q_{k-2, k^{\prime}}(N-2) v_{k^{\prime}+2}\right]\right]}^{i \text { is a consumer }} \\
& +\frac{1}{2} \overbrace{\left[g+\delta \sum_{k^{\prime}=k-2}^{N-2} Q_{k-2, k^{\prime}}(N-2) v_{k^{\prime}+2}\right]}^{i \text { is a producer }}\} \text {. }
\end{aligned}
$$

If $i$ meets a cooperator (probability $\left.\sigma_{k}\right), Q_{k-1, k^{\prime}}(N-2)$ is the probability that $k^{\prime}$ defectors exit matches between the remaining $k-1$ defectors and $N-k-1$ cooperators. Hence, the number of defectors grows to $k^{\prime}+1$ if $i$ is a consumer and $k^{\prime}+2$ otherwise. If $i$ meets a defector (probability $\left.1-\sigma_{k}\right), Q_{k-2, k^{\prime}}(N-2)$ is the probability that $k^{\prime}$ defectors exit meetings between the remaining $k-2$ defectors and $N-k$ cooperators. Hence, next round there are $k^{\prime}+2$ defectors, independent of the role assigned to $i$. 


\section{The main result}

Proposition 1. There exists $0<\delta_{1}<\delta_{2} \leq 1$ such that for $\delta \in\left[\delta_{1}, \delta_{2}\right] \cap(0,1)$ the strategy in Definition 1 supports full cooperation as a sequential equilibrium.

To prove it we establish that: there exists $\delta_{1} \in(0,1)$ such that equilibrium deviations are suboptimal for $\delta \geq \delta_{1}$; there exists $\delta_{2} \in(0,1]$ such that off-equilibrium punishment is incentive compatible for $\delta \leq \delta_{2} ; \delta_{1}<\delta_{2}$.

Equilibrium deviations: A producer who deviates in equilibrium gains $g$ and has continuation payoff $v_{2}$. Using (3), his payoff is

$$
v_{1}^{p}=g+\delta v_{2}
$$

Using $v_{0}^{p}$ from (1), deviating is suboptimal if

$$
v_{1}^{p} \leq v_{0}^{p} \quad \Leftrightarrow \quad g+\delta v_{2} \leq \frac{\delta}{2(1-\delta)}
$$

Using (3):

$$
\frac{g}{1+l} \leq \frac{\delta}{2(1-\delta)}\left[\frac{1-g+l}{1+l}-\phi_{2}(\delta)\right]
$$

From Lemma 2, rewrite (4) as a function of $\phi_{1}(\delta)$ :

$$
\frac{1-g+l}{1+l} \leq \phi_{1}(\delta)
$$

$\phi_{1}$ maps $[0,1)$ into $(0,1]$ and it is a continuous, decreasing function of $\delta$ (Lemma 1). Then, it is invertible, i.e., $\delta=\phi_{1}^{-1}(x)$ for $x \in(0,1]$. Letting $\alpha:=\frac{g}{1+l} \in(0,1)$, there exists

$$
\delta_{1}:=\phi_{1}^{-1}(1-\alpha) \in(0,1)
$$


Monotonicity of $\phi_{1}$ ensures that for all $\delta \in\left[\delta_{1}, 1\right)$ expression (4) is satisfied and equilibrium deviations are suboptimal.

Off-equilibrium deviations: Suppose there are $k$ defectors and let player $i$ be one of them. Suppose this player, as a producer, deviates from the action prescribed by the punishment strategy, cooperating instead of defecting, and will follow the punishment strategy thereafter. The payoff to player $i$ is:

$$
\begin{aligned}
& \tilde{v}_{k}=\sigma_{k}\{\frac{1}{2} \overbrace{\left[1+\delta \sum_{k^{\prime}=k-1}^{N-2} Q_{k-1, k^{\prime}}(N-2) v_{k^{\prime}+1}\right]}^{i \text { is a consumer }}+\frac{1}{2} \overbrace{\left[0+\delta \sum_{k^{\prime}=k-1}^{N-2} Q_{k-1, k^{\prime}}(N-2) v_{k^{\prime}+1}\right]}^{i \text { is a producer }}\} \\
& +\left(1-\sigma_{k}\right)\{\frac{1}{2} \overbrace{\left[-l+\delta \sum_{k^{\prime}=k-2}^{N-2} Q_{k-2, k^{\prime}}(N-2) v_{k^{\prime}+2}\right]}^{i \text { is a consumer }} \\
& +\frac{1}{2} \overbrace{\left[0+\delta \sum_{k^{\prime}=k-2}^{N-2} Q_{k-2, k^{\prime}}(N-2) v_{k^{\prime}+2}\right]}^{i \text { is a producer }}\} \text {. }
\end{aligned}
$$

In the first line the deviator meets a cooperator (a defector, in the second line). Comparing $\tilde{v}_{k}$ to $v_{k}$ we see that $g$ is replaced by 0 when meeting a consumer, while $v_{k^{\prime}+2}$ is replaced by $v_{k^{\prime}+1}$ when meeting a cooperating consumer.

Deviating by choosing $C$ instead of $D$ off equilibrium is suboptimal if $\tilde{v}_{k} \leq v_{k}$, for all $k \geq 2$, i.e.,

$$
\sigma_{k} \delta \sum_{k^{\prime}=k-1}^{N-2} Q_{k-1, k^{\prime}}(N-2)\left[v_{k^{\prime}+1}-v_{k^{\prime}+2}\right] \leq g, \quad k=2, \ldots, N
$$

The left-hand side represents the expected gain from slowing down the contagious defection process; the right-hand side represents the expected loss. Using (3), 
expression (7) becomes

$$
\frac{\sigma_{k} \delta}{2(1-\delta)} \sum_{k^{\prime}=k-1}^{N-2} Q_{k-1, k^{\prime}}(N-2)\left[\phi_{k^{\prime}+1}(\delta)-\phi_{k^{\prime}+2}(\delta)\right] \leq \frac{g}{1+l}, \quad k=2, \ldots, N
$$

Since $\sigma_{k}$ and $\phi_{k}(\delta)-\phi_{k+1}(\delta)$ fall in $k$ (Camera and Gioffré, 2014, Theorem 2), the most stringent case is $k=2$. Given the first row of $\mathcal{Q}_{M}$ (with $M=N-2$ ), we need

$$
\frac{\sigma_{2} \delta}{2(1-\delta)}\left\{Q_{11}(N-2)\left[\phi_{2}(\delta)-\phi_{3}(\delta)\right]+Q_{12}(N-2)\left[\phi_{3}(\delta)-\phi_{4}(\delta)\right]\right\} \leq \alpha
$$

For each $\alpha \in(0,1)$, expression (8) always holds if $\delta$ is sufficiently small. To see why, notice that the left-hand side of (8) is an increasing, continuous function of $\delta \in[0,1)$ and it is equal to zero if $\delta=0 .{ }^{3}$ Let $\delta_{2}$ be the maximum value of $\delta \in(0,1]$ such that (8) holds. By continuity, this upper-bound of $\delta$ exists for each $\alpha \in(0,1)$. Therefore, deviations off equilibrium are suboptimal for all $\delta \in\left[0, \delta_{2}\right] \cap(0,1)$.

To conclude the proof of Proposition 1, we need to prove that the interval $\left[\delta_{1}, \delta_{2}\right]$ is well-defined for each $\alpha \in(0,1)$.

Lemma 3. We have $\delta_{1}<\delta_{2}$ for each $\alpha \in(0,1)$.

Proof. In Appendix.

${ }^{3}$ The monotonicity of $\frac{\phi_{k}(\delta)-\phi_{k+1}(\delta)}{1-\delta}$ follows from Camera and Gioffré (2014, Theorem 2). 


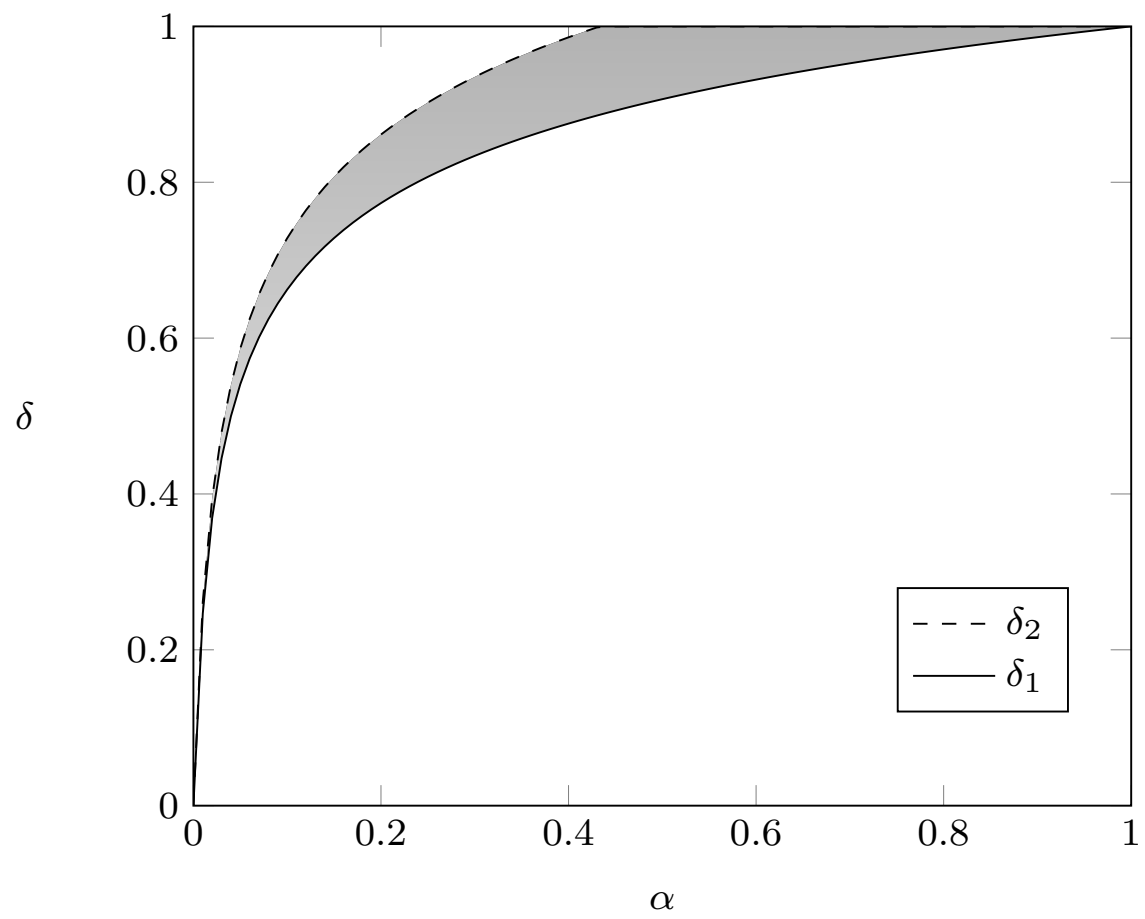

Figure 3: Discount factor thresholds $\delta_{1}$ and $\delta_{2}$ for $N=20$ and $\alpha \in(0,1)$.

The Lemma reveals that for each $\alpha \in(0,1)$ there always exists an interval $\left[\delta_{1}, \delta_{2}\right] \cap(0,1)$ such that deviations in and off equilibrium are suboptimal for all $\delta \in\left[\delta_{1}, \delta_{2}\right] \cap(0,1)$. Figure 3 displays how the interval $\left[\delta_{1}, \delta_{2}\right]$ changes with $\alpha$ when we fix $N=20$, i.e., when there are 10 pairs playing a helping game in each round. The shaded area includes all the points $(\alpha, \delta)$ that support full cooperation as a sequential equilibrium under private monitoring, using the contagious punishment described in Definition 1.

\section{An application}

This section shows how to apply the technique we have developed, in order to determine the threshold discount factors $\delta_{1}$ and $\delta_{2}$ to a pre-existing experimental design. Specifically, we consider the design in Camera and Casari (2014), with 
four players, and in Camera et al. (2013), where there are groups of $N=4,8,32$ players. In those studies, the payoff from cooperation to a consumer and producer are $a=20$ and $b=2$, while the payoff from defection are $x=y=8$ for each consumer and producer. To map those payoffs into the ones described in Table 1 we normalize all payoffs $a, b, x, y$ first by subtracting $b$ and then dividing by $a-b$. We thus have the two cooperation payoffs $(a-b) /(a-b)=1$ and $(b-b) /(a-b)=0$, and the two defection payoffs $-l=(x-b) /(a-b)=1 / 3$, and $g=(y-b) /(a-b)=1 / 3$. In this case $\alpha=0.5$. Those games both assume $\delta=0.93$.

The threshold discount factors. Suppose that subjects follow a grim strategy of community punishment as describe in Definition 1. That is to say, we conjecture that subjects react to defections that they have directly experienced in the game, but not to defections publicly observed in some other match. In this case, Figure 4 reports the exact values of $\delta_{1}$ and $\delta_{2}$ for the groups of $N=4,8,32$. These have been calculated using Matlab code reported in Appendix. 


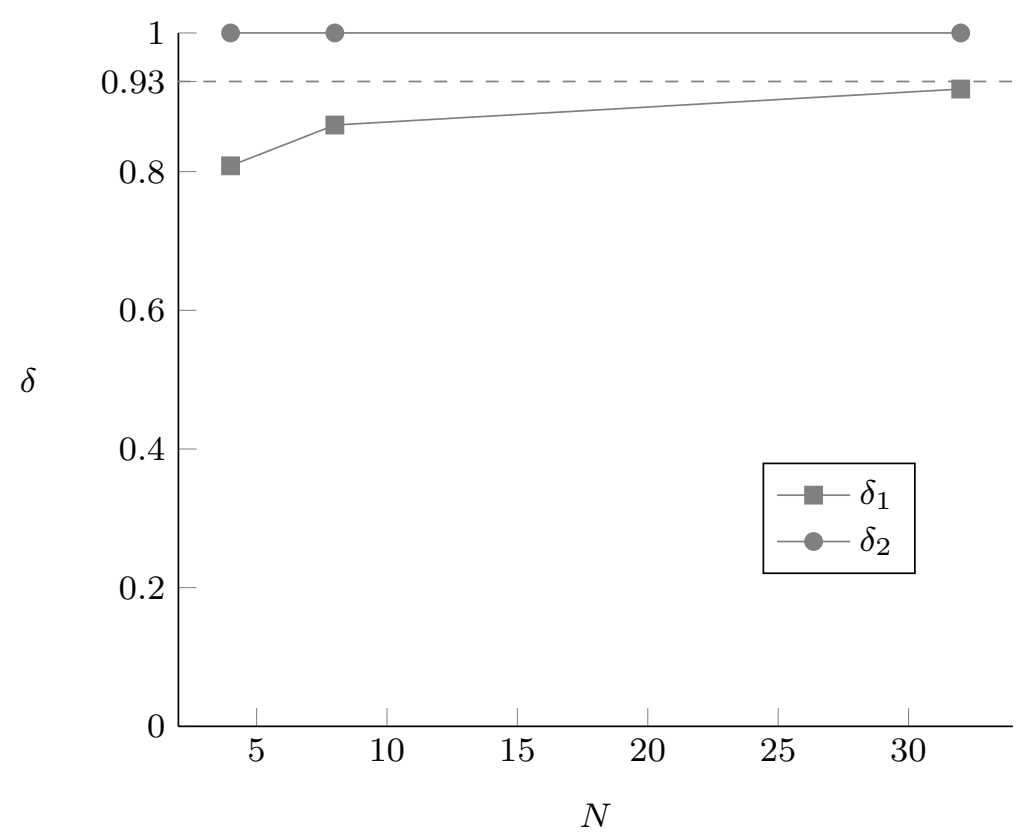

Figure 4: Thresholds $\delta_{1}$ and $\delta_{2}$ for $N=4,8,32$ and $\alpha=0.5$.

Notes: Here $l=-1 / 3, g=1 / 3$ so $\alpha=1 / 2$. These parameters correspond to the experimental parameters in Camera and Casari (2014) and Camera et al. (2013). For $N=4$ the figure identifies the threshold discount factors in Camera and Casari (2014). For $N \geq 4$, it identifies the threshold discount factors in Camera et al. (2013) (where there is anonymous public monitoring) under the conjecture that subjects do not trigger based on publicly available information, but instead follow a grim strategy of community punishment; this is equivalent to conjecturing that subjects react to defections that they have directly experienced in the game, but not to defections publicly observed in some other match.

The value $\delta_{2}=1$ for all group sizes. This means that in all those cases players have an incentive to punish, off equilibrium. Instead, the value $\delta_{1}<1$ for all group sizes and it increases as the group size increases. That is to say, the incentive to defect in equilibrium instead of cooperating decreases as the size of the economy grows. In Camera and Casari (2014) and Camera et al. (2013) we have $\delta=0.93$, therefore a social norm of community punishment can theoretically support full cooperation for all group sizes $N \leq 32$ : we have approximately $\delta_{1}=0.81,0.87,0.92$ for $N=4,8,32$ respectively.

This does not mean that players may not be able to support cooperation 
through some other means, of course. For example, in Camera et al. (2013) there is public monitoring so players could adopt a social norm that triggers punishment based on observation of defections anywhere in the group. ${ }^{4}$ What we are doing here is simply identifying if conditions exist that support full cooperation as a sequential equilibrium under the conjecture that subjects react to defections that they have directly experienced in the game, but choose to ignore defections that have occurred in some other match - even if these defections have been made public. Or, equivalently, for a design in which there is private monitoring. The calculations reveal that we do not theoretically need public monitoring to support full cooperation even with 32 subjects.

\section{The decline in cooperation under the norm of community punishment.}

Figure 5 traces the share of players who are in a cooperative state following an initial defection, under the conjecture that everyone follows the grim strategy in Definition 1. These are not necessarily producers, because roles in each round are assigned at random, with a coin flip. These are simply players who have seen (or done) a choice $D$ in one or more of the previous rounds. Hence, they should choose $D$ this round if they are randomly assigned the role of producer.

The figure shows that by round 10 we effectively expect to see no cooperator if the group is of size $N=4,8$. This happens by round 15 in groups of size $N=32$. The implication is that in the laboratory, if subjects adopt a grim strategy as in Definition 1, then we should observe a cooperation rate close to zero, about 10

\footnotetext{
${ }^{4}$ This supports full cooperation as an equilibrium for all group sizes as long as $\delta \geq 2 / 3$; see (Camera et al., 2013, Supplementary Information).
} 
rounds after the initial defection in groups of four players. Below, we visually compare this theoretical prediction with data from the experiment in Camera and Casari (2014), which is based on two sessions with groups of $N=4$ players, under private monitoring. This can be informative about whether or not subjects developed of a social norm of community punishment.

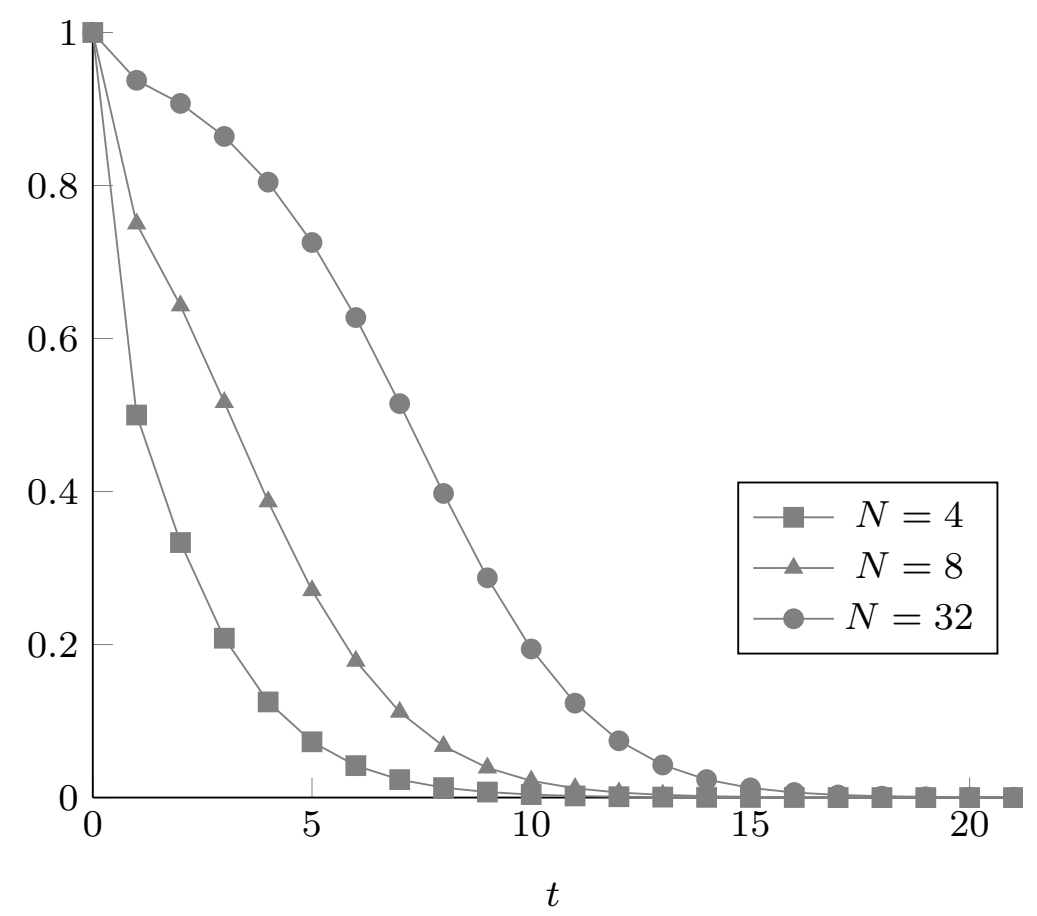

Figure 5: Expected share of players in a cooperative state at the start of a round.

Notes: The figure calculates the expected share of players in a cooperative state at the start of a round $t \geq 1$, given that someone moved off equilibrium in round $t=0$. This is derived under the conjecture that subjects follow a grim strategy of community punishment; this is equivalent to conjecturing that subjects react to defections that they have directly experienced in the game, but not to defections that might have occurred in some other match, even if publicly observed. Here $t=1$ refers to the round immediately after a player moved off-equilibrium by defecting. Hence, in $t=1$ two players are in a punishment state. The share of cooperators in a group with $N$ players is $1-d / N$ where $d$ is the number of defectors at the start of the period.

Realized cooperation in the lab. We start by reporting some statistics about the Baseline treatment in Camera and Casari (2014) where the group size was $N=4$ and there was private monitoring; the data are taken from that experi- 
ment and discussed in the associated article. There were two sessions each with twenty subjects. Each session consisted of five consecutive supergames of uncertain duration. In each supergame subjects were arranged into five groups of four individuals, using strangers rematching after each supergame. The uncertain duration was implemented by a random device that was operated during the experiment. The computer program was calibrated so that there was a $93 \%$ probability of continuation and a $7 \%$ probability of termination after each round, starting from the first round of play. That is to say, $\delta=0.93$ if we consider players as being risk neutral. The realized length of supergames varied greatly in the experiment, going from a minimum of 2 rounds to a maximum of 26 rounds. ${ }^{5}$

In the experiment many subjects defected in round 1. The left panel in Figure 6 reports the average cooperation rate in a group in round 1, for 50 supergames pooled as follows: supergames 1-5 all together, and the last supergame 5 by itself. The figure suggests that the average group starts with at least one producer defecting so cooperation is partial from the start. Recall that in each group there are two producers who take an initial action so when we further study groups we see that both initial producers defected in 28 percent of groups, one producer defected in 42 percent of groups and only 30 percent of groups had full cooperation initially. Given that someone defects in round 1 of a supergame, we should see punishment starting in the second round if players adopted the grim strategy, in 70 percent of the groups. The theory developed above suggests that if subjects adopted a norm of community punishment as in Definition 1, then the contagious

\footnotetext{
${ }^{5}$ For additional details about this experiment see the discussion in Camera and Casari (2014) and supplementary information.
} 
punishment process starts immediately in the round following the defection and leads to a very rapid decline in cooperation. We expect that full defection should emerge by round 10, as reported in Figure 5.
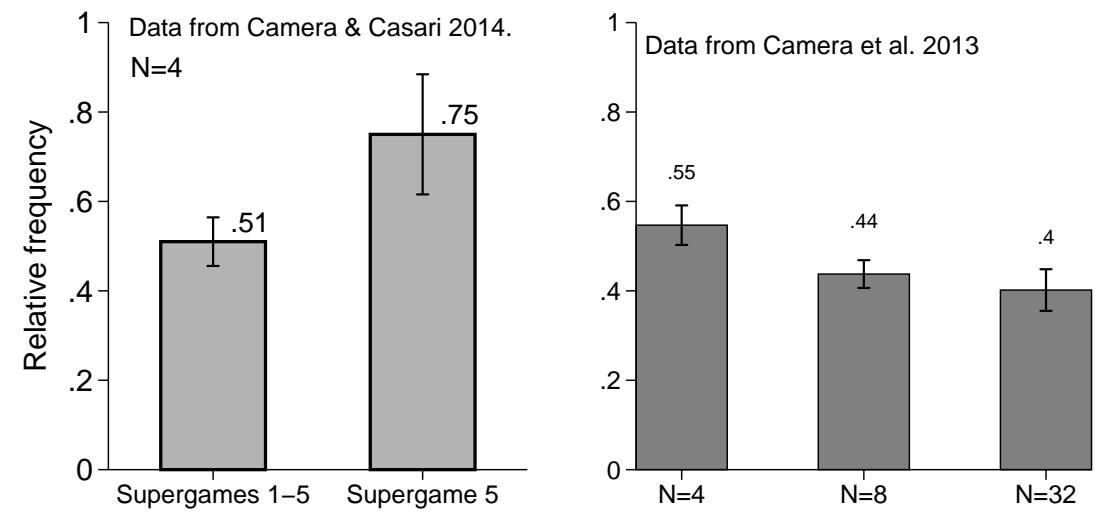

Figure 6: Cooperation in round 1 for $N=4$ (left panel) and $N \geq 4$ (right panel).

Notes: Left panel: data from baseline treatment in Camera and Casari (2014); average realized cooperation rate in a group of four subjects, in the first round of play, with standard error bands. We pool together all groups in supergames 1-5 (50 obs.), and, separately, all groups in supergame 5 (10 obs.). Right panel: data from Control condition in Camera et al. (2013); average realized cooperation rate in a group of $\mathrm{N}=4,8,32$ in the first round of play, with standard error bands. We pool together all same-size groups in supergames 1-4 (64 obs., per size $\mathrm{N}=4$ and $\mathrm{N}=8$ ), and, separately, all 32-player groups in supergame 5 (7 obs.).

To investigate this, consider the dynamics of cooperation in Fig. 7. The left panel reports cooperation rates in the average private monitoring group of Camera and Casari (2014), by round of play, for all supergames 1-5 pooled together. The bands refer to the mean standard error. The maximum duration of a supergame was 26 rounds, with periods 1-20 capturing $91 \%$ of all observations; the expected number of rounds was 14 . Cooperation appears to be volatile. However, it does not permanently decline until after round 20, and does not reach zero. Average cooperation was 0.46 in rounds $1-13$ and 0.38 in the following rounds. This decline is slow as compared to what predicted by the grim strategy, according to which 
by round 10 we expect cooperation close to zero. Moreover, average realized cooperation never hits zero. This suggests that subjects did not coordinate on the norm of community punishment discussed in this paper. Further evidence comes from individual-level observations: about 27 percent of players chose $C$ every time they were producers in a supergame, while about 30 percent always defected in the supergame. Although "Always $C$ " could be consistent with grim play if these subjects did not observe any defection, "Always $D$ " is not.
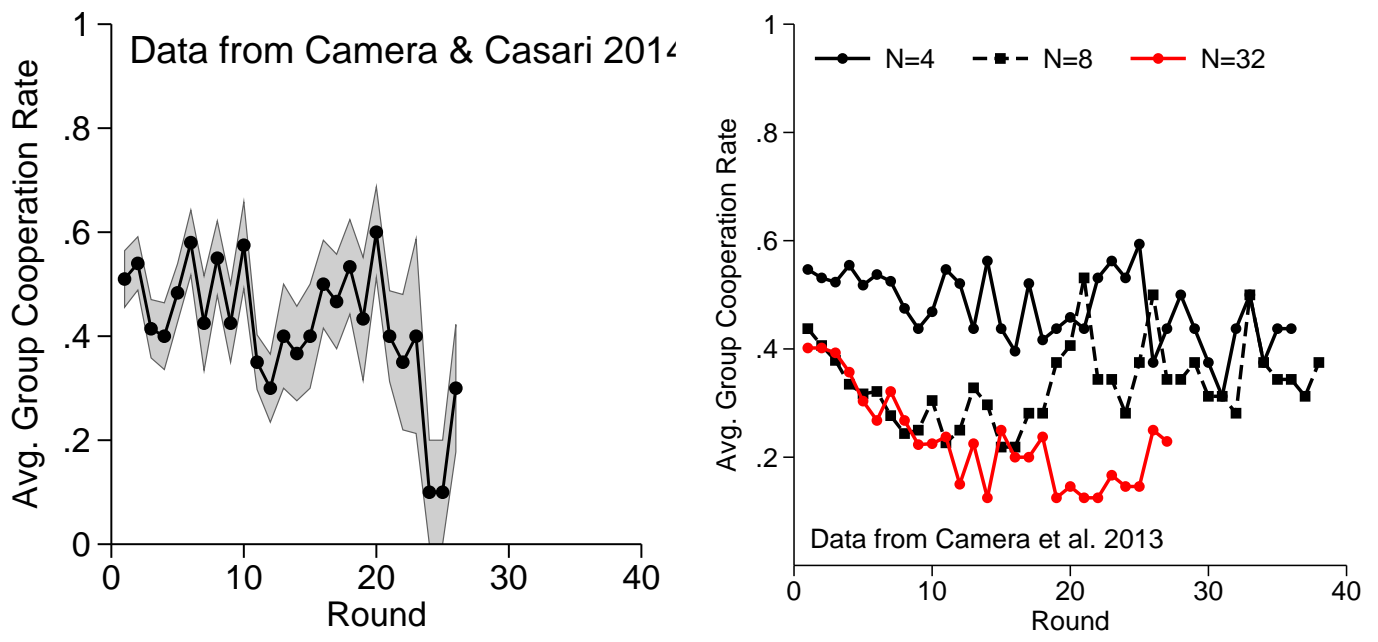

Figure 7: Average realized cooperation for $N=4$.

Notes: Left panel: data from baseline treatment in Camera and Casari (2014). Right panel: data from control condition in Camera et al. (2013). See notes to Fig. 7.

It can be especially helpful to compare the results in the $N=4$ player groups under private monitoring, with results from large groups that can exploit a form of anonymous public monitoring, i.e., a situation in which defections are immediately detected by everyone in the economy. This is interesting for three reasons. First, cooperation should be easier to sustain as compared to private monitoring because a new strategy becomes available: any defection observed anywhere in the group 
can be immediately punished by the entire group (the strategy we study in this paper, clearly, remains available). This implies that the threshold lower bound for the discount factor should be lower. Second, the lowerbound $\delta$ threshold in this case is independent of the size of the economy $N$. Third, a defection should lead to a more rapid drop in cooperation since everyone can see it, while under private monitoring this is impossible.

To do so we use the sessions of the Control condition in Camera et al. (2013). In that experiment, there were different group sizes $N=4,8,32$ and all players in the group were informed about the frequency of $C$ and $D$ in their group, at the end of a round of play, without seeing the identity of actors (anonymous public monitoring). Each session consisted of five consecutive supergames of uncertain duration, with strangers rematching after each supergame. The uncertain duration was again implemented with $\delta=0.93$ but randomization started on round 3 , so players had three rounds for sure. There were five sessions each of which had size $N=32$ in the last supergame, but that differed in the size $N$ in supergames 1-4: in two sessions $N=4$, in two other $N=8$, while in one $N=2$. The payoff matrix was identical to the one in Camera and Casari (2014). ${ }^{6}$ This is why we have separately reported supergame 5 in the left panel of Fig. 6.

The right panel of Fig. 6 reports cooperation in round 1, by group size. Again we see that many subjects defected in round 1 . The presence of public monitoring raised a bit cooperation when we compare similarly-sized groups in the left panel $(N=4)$, which is consistent with the lower threshold lowerbound $\delta$ under public

\footnotetext{
${ }^{6}$ For additional details about this experiment see the discussion in Camera et al. (2013) and
} supplementary information. 
monitoring. However, we also see that defections increase with the size of the group, which is not what is predicted by theory since the size does not affect thresholds under public monitoring. A possible explanation is that larger groups entail greater strategic uncertainty, which leads to lower cooperation in round 1 and subsequent, a point that is discussed in Bigoni et al. (2019). Note that $N=4$ and $N=8$ comprise data from supergames $1-4$, while $N=32$ only from supergame 5; this allows a direct comparison with rates in the left panel. If subjects used a grim punishment we should see a drop in cooperation to zero even faster than in the private monitoring case of the left panel.

The right panel in Fig. 7 refers to data from Camera et al. (2013) separately for each group size $N=4,8,32$. We do not draw the mean standard error bands to avoid cluttering the figure. Given anonymous public monitoring, we expect a sharper and quicker decline in cooperation as compared to the left panel where monitoring is private. Yet, the decline is slow as compared to what predicted by the grim strategy and average realized cooperation never hits zero. Again, these observations are inconsistent with grim play. To address these observations we develop a simple behavioral model that can generate partial cooperation as an equilibrium.

Equilibria with Partial Cooperation: a Behavioral Theory. We develop a simple behavioral model capable of explaining why in the laboratory groups managed to sustain partial cooperation, with some players fully defecting and others fully cooperating. For analytical simplicity and to enhance transparency, 
we work with a large group of players of unit measure. Assume that players can be of two types, rational or behavioral, in proportion $(1-\tau) r$ and $1-(1-\tau) r$, respectively. The distribution of these types is known to all.

Rational players choose between two strategies, always defect (AD) and grim $(\mathrm{G})$, to maximize their payoff. Behavioral players, instead, follow a fixed rule of conduct that does not necessarily maximize an objective function. Some of these players are altruists, who always cooperate; their population measure is $\tau$. These players have a strong pro-social element in their preferences that dominates other purely economic considerations, so they cooperate with everyone independently of the history of play. ${ }^{7}$ The rest, i.e., the population proportion $(1-\tau)(1-r)$, are timid punishers; as producers, they cooperate as long as they have never seen a defection, and otherwise they cooperate with probability $q$ forever after. They do so because they are uncomfortable defecting every time they are producers, as doing so would surely punish innocent behavioral players. This random punishment introduces a noise factor.

The presence of behavioral players raises the incentive to choose $\mathrm{AD}$ for a rational player as compared to the case in which all players are rational studied in Section 2. If all rational players follow $\mathrm{G}$, then full cooperation results and equilibrium payoffs are identical to those earlier reported. Instead, if rational players choose $\mathrm{AD}$, then expected payoffs will differ from those reported in Section 4. This model supports partial cooperation if it is individually optimal for rational players to select $\mathrm{AD}$ instead of $\mathrm{G}$. To make this point we must calculate payoffs

\footnotetext{
${ }^{7}$ The strength of this pro-sociality may depend on social distance as measured by the size of the group. For example, one could thus imagine that $\tau$ decreases with the group size.
} 
off-equilibrium. Below, we do so focusing on the least favorable case for AD to be selected, which corresponds to a public monitoring game because it gives a smaller incentive to choose $D$ as compared to the case of private monitoring because in the latter case defections are revealed more slowly, over time, through interaction. Instead, with public monitoring there is an immediate reaction to the defection by the entire economy. This also simplifies the analysis because AD corresponds to the punishment mode of G, so we do not have to worry about the complicated transition dynamics discuss in Section 3, to make our point.

In fully cooperative equilibrium all rational players selected G. Here everyone plays $C$, hence the payoff to any producer is $v_{0}^{p}=\frac{\delta}{2(1-\delta)}$ as seen earlier. Suppose a rational player, who is a producer, considers deviating from $\mathrm{G}$ to $\mathrm{AD}$ in fully cooperative equilibrium. The payoff to this deviator is

$$
\hat{v}^{p}:=g+\delta \hat{v}
$$

where $\hat{v}:=\frac{\hat{v}^{p}+\hat{v}^{c}}{2}$, is the expected continuation payoff off-equilibrium since the player has an equal chance to be producer or consumer next round. Here,

$$
\hat{v}^{c}:=\tau+(1-\tau)\{r(-l)+(1-r)[q+(1-q)(-l)]\}+\delta \hat{v}
$$

defines the off-equilibrium payoff to the deviator, if he is a consumer.

Using $\hat{v}^{p}$ and $\hat{v}^{c}$ we obtain:

$$
\hat{v}=\frac{g+\tau+(1-\tau)[-l+(1-r) q(1+l)]}{2(1-\delta)}
$$


Deviating from $\mathrm{G}$ to $\mathrm{AD}$ is optimal in cooperative equilibrium if

$$
v_{0}^{p} \leq \hat{v}^{p} \quad \Leftrightarrow \quad \delta \leq \bar{\delta}:=\frac{2 g}{1+g-\tau-(1-\tau)[-l+(1-r) q(1+l)]}
$$

Depending on the parameters of the game and the distribution of types, we may have $\delta \leq \bar{\delta}$. If so, there will be partial cooperation because only behavioral players cooperate, while rational players will always defect. In fact, $\bar{\delta}>1$ is possible, in which case $\mathrm{AD}$ is always the optimal choice for a rational player.

Below we demonstrate, using realized choice data from the experiment in Camera and Casari (2014), that $\delta \leq \bar{\delta}$, i.e., the partial cooperation observe in the data is roughly consistent with the simple theory developed here.

To start, calculate the number of altruists in the data; approximately 27 percent of players chose $C$ every time they were producers in a supergame, hence set $\tau=0.27$. Approximately 30 percent of players selected $D$ in all rounds of a supergame in which they were producers. Using the theory developed above, we conjecture that these are all rational players who optimally selected AD because $\delta \leq \bar{\delta}$. If so, then $0.3=(1-\tau) r$, so $r=0.41$ approximately. Finally, the remaining 43 percent of players cooperated partially, i.e., they are the timid punishers. The data reveals that these players' average cooperation rate is 0.47 , so we set $q=0.47$. Substituting these value and $g=-l=1 / 3$ (as discussed in Section 5 ) in $\bar{\delta}$ we obtain $\bar{\delta}=0.97$ approximately, which confirms the conjecture $\delta=0.93 \leq \bar{\delta}$. Hence, the simple behavioral theory reported in this section could offer a possible explanation for the partial cooperation observed in the experiment. 


\section{Discussion}

Recent experiments have investigated cooperation and the emergence of fiat monetary systems in groups of strangers who face one-sided "helping games," under repeated random assignment of roles and opponents (Camera et al., 2013; Camera and Casari, 2014). This study derived a general proof of existence and characterization of cooperative equilibrium when there is private monitoring. This generalizes the study of social norms to random matching games other than the standard Prisoner's Dilemma. The analysis shows how to calculate the threshold discount factors necessary to support full cooperation, for any payoff matrix and group size; Matlab codes to perform these calculations are provided in the Supplementary Materials published online.

The analysis in this paper has general applicability. It can be adapted to study games where more than one player makes a choice in each round. For example, the study in Camera and Gioffré (2018) uses this technique in the infinitely repeated Prisoner's Dilemma to determine strategies that can support efficient play when players are "too patient" and therefore the standard grim community punishment norm is ineffective. This same technique can be applied to study public goods games, as well as games with asymmetric payoff matrices.

In this paper, we have used this technique to simulate the spread of punishment predicted by the model and then visually compared it to punishments observed in the laboratory. This rough comparison reveals a mismatch between theory and data, suggesting that grim community punishments are not the norm in the laboratory. This hypothesis can be tested through a formal estimation of the 
strategies adopted in indefinitely repeated helping games. A way to proceed could be to adapt the technique of strategy estimation of PD games discussed in Dal Bó and Fréchette (2018), for fixed pairs, and in Camera et al. (2012) for random matching economies of strangers, to the helping game setting we studied here.

Camera et al. (2012) can offer some insight into the strategies adopted in repeated helping games because it estimates strategies in groups of strangers based on a design having many similarities with that in Camera and Casari (2014) discussed in Section 5. The main difference is the use of a PD game as opposed to the one-sided helping game, different payoffs from cooperation, and a slightly higher continuation probability (0.95 instead of 0.93$)$. The study reports an average cooperation rate in the first round of a supergame of 67 percent, while the overall cooperation rate was about 54 percent, numbers that are in line with what we saw in Section 5. Econometric analysis reveals that only one out of four individuals exhibits behavior that is consistent with the use of the grim trigger strategy. Subjects showed a tendency to tolerate defections and, as a result, systematic defectors and systematic cooperators often coexisted in a group much as it happened in the repeated helping games discussed above. This lends further support to the notion that norms of cooperation based on a grim community punishment scheme, although theoretically appealing, are uncommon in practice. To explain the data the theory could be adjusted to incorporate behavioral elements. We have done so by surmising that at least some individuals act based upon a social component in their preferences, while others may be hesitant to punish a defection to avoid affecting innocent individuals. 


\section{References}

Bigoni, M., G. Camera, and M. Casari. "Cooperation among strangers with and without a monetary system." Forthcoming in C. Monica Capra, Rachel Croson, Mary Rigdon and Tanya Rosenblat (eds), Handbook of Experimental Game Theory, Cheltenham, UK and Northampton, MA, USA: Edward Elgar Publishing.

Bigoni, M., Camera, G., and M. Casari. (2019). Partners or Strangers? Cooperation, monetary trade, and the choice of scale of interaction. American Economic Journal: Microeconomics 11(2), 1-34.

Camera, G., and M. Casari. (2009). Cooperation among strangers under the shadow of the future. American Economic Review, 99 (3), 979-1005.

Camera, G., M. Casari, and M. Bigoni. (2012). Cooperative strategies in anonymous economies: an experiment. Games and Economic Behavior, 75, 570-586.

Camera, G., M. Casari, and M. Bigoni. (2013). Money and trust among strangers. Proceedings of the National Academy of Sciences, 110 (37), 14889-14893.

Camera, G., and M. Casari. (2014). The Coordination Value of Monetary Exchange: Experimental Evidence. American Economic Journal: Microeconomics, $6(1), 290-314$.

Camera, G., and A. Gioffré. (2014). A tractable analysis of contagious equilibria. Journal of Mathematical Economics 50, 290-300.

Camera, G., and A. Gioffré. (2018). Beyond Grim: Punishment Norms in the Theory of Cooperation. Working paper, Uiversity of Florence. 
Dal Bó, P. and G. Fréchette. (2018). On the determinants of cooperation in infinitely repeated games: A survey. Journal of Economic Literature, 56 (1), 60114.

Duffy, J., and J. Ochs. (2009). Cooperative behavior and the frequency of social interaction. Games and Economic Behavior, 66(2), 785-812.

Ellison, G. (1994). Cooperation in the prisoner's dilemma with anonymous random matching. Review of Economic Studies 61, 567-88.

Kandori, M. (1992). Social norms and community enforcement. Review of Economic Studies 59, 63-80.

Nowak, M.A. (2006). Five rules for the evolution of cooperation. Science $314(5805), 1560-1563$ 


\section{A Appendix}

\section{A.1 The Helping Game}

Here, we describe in a bit more detail the helping game adopted in this paper, drawing from the discussion presented in Bigoni et al. (forthcoming). Considering one round of play, the helping game is a cooperative task that involves two players but is one-sided, i.e., one player is active and the other passive in the round. The producer and consumer roles are assigned at random as soon as a pair is formed in the period. We call the active player "producer" and the passive one "consumer." Since the game is one-sided, technically speaking it is a decision problem, similar to a dictator's game.

One can think of the producer as being endowed with a good at the start of the round, while the consumer has no endowment at all. Alternatively, one can think of the producer as being able to make a gift to the consumer ("help"). Either way, the point is that both would like to consume something but the consumer has a greater utility from doing so in that round as compared to the producer. For this reason, the producer is given the option to unilaterally transfer the good to the consumer. If this occurs, then we say that the producer cooperates or, equivalently, "helps" the consumer. The other alternative for the producer is to consume the good himself, or defect or, yet equivalently, to refuse to help the consumer. This decisional situation is described in Table A1, below.

Table A1: The Helping Game.

\begin{tabular}{lcc} 
& \multicolumn{2}{c}{ Producer's choice } \\
& $H e l p(C)$ & Do not help $(D)$ \\
\cline { 2 - 3 } Payoff to producer & $b$ & $y$ \\
Payoff to consumer & $a$ & $x$
\end{tabular}

Since the producer is assumed to value consumption we have $y>b$, i.e., the producer prefers to eat his good instead of giving it away. Since the consumer is assumed to derive a greater benefit than the producer from consuming the good, we also have $a>y$. A central feature of the game is that the consumer's net benefit from eating is so large that cooperation is socially efficient, i.e., $a+b>x+y$. In the theory section, we have normalized the cooperation payoff to the consumer and the producer to 1 and 0 , respectively, which gives us a defection payoff of $-l=(x-b) /(a-b)$ to the consumer and of $g=(y-b) /(a-b)$ to the producer (Figure 1). In Section 5, instead, we have used the payoff matrix in Table A1, with $a=20, b=2$, and $x=y=8$. Repeating this decisional situation indefinitely, with random counterparts, generates a proper game and is necessary (but not sufficient) to create dynamic incentives for cooperation. 


\section{Proof of Lemma 1}

The derivative of $\phi_{k}(\delta)$, for $k=1, \ldots, N-1$ is

$$
\phi_{k}^{\prime}(\delta)=-e_{k}^{\top}\left(\mathcal{I}_{N}-\delta \mathcal{Q}_{N}\right)^{-1}\left[\mathcal{I}_{N}-(1-\delta) \mathcal{Q}_{N}\left(\mathcal{I}_{N}-\delta \mathcal{Q}_{N}\right)^{-1}\right] \sigma
$$

where we have used $\frac{d \mathcal{A}^{-1}(\delta)}{d \delta}=-\mathcal{A}^{-1}(\delta) \frac{d \mathcal{A}(\delta)}{d \delta} \mathcal{A}^{-1}(\delta)$, with $\mathcal{A}(\delta):=\mathcal{I}_{N}-\delta \mathcal{Q}_{N}$. To prove that $\phi_{k}^{\prime}(\delta)<0$ if $k<N$, notice that $(1-\delta)\left(\mathcal{I}_{N}-\delta \mathcal{Q}_{N}\right)^{-1} \sigma \leq \sigma$ (with strict inequality for $k \leq N-1)$ since $(1-\delta)\left(\mathcal{I}_{N}-\delta \mathcal{Q}_{N}\right)^{-1} \mathbf{1}=\mathbf{1}$ and $\sigma_{k} \in \sigma$ decreases in $k$. Therefore, $\mathcal{Q}_{N}(1-\delta)\left(\mathcal{I}_{N}-\delta \mathcal{Q}_{N}\right)^{-1} \sigma \leq \mathcal{Q}_{N} \sigma \leq \mathcal{I}_{N} \sigma$. So, we have

$$
\left(\mathcal{I}_{N}-\delta \mathcal{Q}_{N}\right)^{-1}(1-\delta) \mathcal{Q}_{N}\left(\mathcal{I}_{N}-\delta \mathcal{Q}_{N}\right)^{-1} \sigma \leq\left(\mathcal{I}_{N}-\delta \mathcal{Q}_{N}\right)^{-1} \sigma
$$

which for $k=1, \ldots, N-1$ holds with strict inequality.

\section{Proof of Lemma 2}

We have

$$
v_{1}=\frac{1+g}{2}+\delta Q_{11}(N) v_{1}+\delta Q_{12}(N) v_{2},
$$

which, using (3) and rearranging, becomes

$$
\left[1-\delta Q_{11}(N)\right]\left[\frac{g-l}{2(1-\delta)}+\frac{1+l}{2(1-\delta)} \phi_{1}(\delta)\right]=\frac{1+g}{2}+\delta Q_{12}(N)\left[\frac{g-l}{2(1-\delta)}+\frac{1+l}{2(1-\delta)} \phi_{2}(\delta)\right]
$$

Rearranging,

$$
\left[1-\delta Q_{11}(N)\right] \frac{\phi_{1}(\delta)}{1-\delta}=1+\delta Q_{12}(N) \frac{\phi_{2}(\delta)}{1-\delta}
$$

Using $Q_{11}(N)+Q_{12}(N)=1$,

$$
\frac{\delta Q_{12}(N)}{1-\delta}\left[\phi_{1}(\delta)-\phi_{2}(\delta)\right]=1-\phi_{1}(\delta)
$$

and note that $Q_{12}(N)=1 / 2$.

\section{Proof of Lemma 3}

It is sufficient to show that there exists a value $\bar{\delta}_{2} \in(0,1]$, with $\bar{\delta}_{2} \leq \delta_{2}$, such that $\delta_{1}<\bar{\delta}_{2}$. Since $\phi_{k}(\delta)-\phi_{k+1}(\delta)$ decreases with $k$, condition (8) holds if

$$
\frac{\sigma_{2} \delta}{2(1-\delta)}\left[\phi_{2}(\delta)-\phi_{3}(\delta)\right] \leq \alpha
$$

which is satisfied if

$$
\frac{\sigma_{2} \delta}{2(1-\delta)}\left[\phi_{1}(\delta)-\phi_{2}(\delta)\right] \leq \alpha
$$


Let $\bar{\delta}_{2}$ be the maximum value of $\delta \in(0,1]$ such that (1) holds. Since (1) implies (8), we have $\bar{\delta}_{2} \leq \delta_{2}$. First we show how to construct $\bar{\delta}_{2}$. Using Lemma 2, (1) is equivalent to

$$
1-\phi_{1}(\delta) \leq \frac{\alpha}{\sigma_{2}} \quad \Leftrightarrow \quad 1-\frac{N-1}{N-2} \alpha \leq \phi_{1}(\delta)
$$

where $\sigma_{2}=\frac{N-2}{N-1}$.

There are two cases. If $\alpha \in\left[\frac{N-2}{N-1}, 1\right)$ then (2) holds for all $\delta \in[0,1)$ since $\phi_{1}(\delta) \geq 0$. Instead, if $\alpha \in\left(0, \frac{N-2}{N-1}\right)$ then the left-hand side of (2) lies in the unit interval. Hence, by continuity of $\phi_{1}$ there exists a value $\bar{\delta}_{2} \in(0,1)$ such that $(2)$ holds with equality. Since $\phi_{1}$ is a decreasing function of $\delta$ we can conclude that (2) is satisfied for all $\delta \in\left(0, \bar{\delta}_{2}\right]$. Hence, deviating by cooperating is suboptimal off equilibrium for all $\delta \in\left(0, \bar{\delta}_{2}\right] \cap(0,1)$, where

$$
\bar{\delta}_{2}:= \begin{cases}\phi_{1}^{-1}\left(1-\frac{N-1}{N-2} \alpha\right) & \text { if } \quad \alpha \in\left(0, \frac{N-2}{N-1}\right) \\ 1 & \text { if } \quad \alpha \in\left[\frac{N-2}{N-1}, 1\right) .\end{cases}
$$

Now, we show that $\delta_{1}<\bar{\delta}_{2}$. For $\alpha \in\left[\frac{N-2}{N-1}, 1\right)$ the proof is obvious since $\bar{\delta}_{2}=1$. For $\alpha \in\left(0, \frac{N-2}{N-1}\right)$, use the definition of $\bar{\delta}_{2}$ and $\delta_{1}$ to derive the inequality

$$
\phi_{1}\left(\bar{\delta}_{2}\right)=1-\frac{N-1}{N-2} \alpha<1-\alpha=\phi_{1}\left(\delta_{1}\right)
$$

Since $\phi_{1}(\delta)$ falls in $\delta \in(0,1)$ then $\phi_{1}\left(\bar{\delta}_{2}\right)<\phi_{1}\left(\delta_{1}\right)$ implies $\bar{\delta}_{2}>\delta_{1}$. This completes the proof. 